\title{
Dietary and lifestyle inflammatory scores and risk of incident diabetes: a prospective cohort among participants of Tehran lipid and glucose study
}

Farshad Teymoori ${ }^{1,2}$, Hossein Farhadnejad ${ }^{1}$, Ebrahim Mokhtari ${ }^{1}$, Mohammad Hassan Sohouli ${ }^{1,2}$, Nazanin Moslehi ${ }^{i^{*}}$, Parvin Mirmiran ${ }^{1 *}$ and Fereidoun Azizi ${ }^{3}$

\begin{abstract}
Background: Inflammation is a precursor of chronic disease, which is affected by lifestyle and dietary habits. Recently empirical dietary inflammatory patterns (EDIP), dietary inflammation scores (DIS), and lifestyle inflammation scores (LIS) were developed to indicate lifestyle and dietary contributions in systemic inflammation. The current study aimed to investigate the associations between these indices and the incidence of diabetes among Tehranian adults.

Methods: A total of 4624 individuals, aged 20-75 years, who were free of diabetes at baseline (2008-2011), were followed for 5.71 years (2014-2017) to ascertain incident diabetes. Dietary intakes were collected at baseline using the food frequency questionnaire. The hazard ratio (HR) of diabetes was calculated by Cox proportional hazards regression across quartiles of EDIP, DIS, and LIS, adjusted for potential confounders.

Results: The mean \pm SD for the age and BMI of the study population ( $45.1 \%$ male) were $40.8 \pm 12.7$ years and $27.1 \pm 4.1 \mathrm{Kg} . \mathrm{m} 2$, respectively. At the end of the follow-up, $329(7.1 \%)$ diabetes cases were identified. In the multivariable-adjusted model, individuals in the highest compared to the lowest quartile of EDIP (HR $=0.83 ; 95 \% \mathrm{Cl}$ : 0.59-1.15, p for trend =0.286), and LIS (HR=2.41; 95\%Cl:1.61-3.60, P for trend $<0.001)$ had increased risk of diabetes. However, no significant associations were found between the score of DIS and diabetes incidents $(H R=$ 0.83; 95\% Cl:0.59-1.15, p for trend = 0.286).
\end{abstract}

Conclusion: Greater adherence to EDIP and LIS scores was associated with a higher risk of diabetes, while no significant association was found between the DIS score and diabetes incident.

Keywords: Diet, Lifestyle, Inflammation, Diabetes

\footnotetext{
*Correspondence: moslehinazanin@yahoo.com; mirmiran@endocrine.ac.ir 'Nutrition and Endocrine Research Center, Research Institute for Endocrine Sciences, Shahid Beheshti University of Medical Sciences, Tehran, Iran Full list of author information is available at the end of the article
} 


\section{Background}

Type 2 diabetes mellitus (T2DM) is a severe lifethreatening health problem characterized by beta-cells dysfunction, insulin resistance, and high blood glucose levels. This metabolic disease has significant adverse effects on quality of life and increases healthcare costs, comorbidities, and mortality [1]. Through the past recent years, the global prevalence of diabetes has faced a considerable increase and is predicted to rise from approximately 463 million in 2019 to 700 million by 2045 [2].

Chronic inflammation is a well-known risk factor related to chronic diseases, including cardiovascular disease, T2DM, and cancers [3]. Previously, studies have observed significantly higher concentrations of inflammatory factors such as C-reactive protein (CRP), Tumor necrosis factor- $\alpha$ (TNF $\alpha)$, and ILs in individuals with chronic diseases [4, 5]. Also, it has been reported that dietary factors such as high intake of saturated fat and low intake of fruit, vegetables, and whole grains, along with other lifestyle factors including obesity, physical inactivity, and cigarette smoking, collectively play an essential role in the estimation of diet and lifestyle influence on systemic inflammation $[6,7]$. So, recently, the researchers sought to determine the inflammation caused by environmental factors such as dietary factors in individuals without directly measuring the serum inflammatory indices and examining its association with the risk of various chronic diseases.

Previously, a pre-defined score called dietary inflammatory index (DII) was developed to assess the contributions of dietary exposures in the body's inflammatory status and consequently the risk of chronic disease development. In this regard, three cross-sectional [8-10] and prospective [11] studies observed a higher DII score positively associated with a higher risk of diabetes. DII estimates the potential relation of selected food parameters with the body's inflammatory status [12]. DII mostly focuses on the anti/pro-inflammatory nutrients without considering the nutrient interactions in body homeostasis and other effects of unmeasured and unknown anti/ pro-inflammatory compounds of whole foods and beverages. Recently two novels inflammatory indices, including empirical dietary inflammatory pattern (EDIP) and dietary inflammation scores (DIS), have been proposed based on the association between food groups and inflammatory markers $[13,14]$.

Furthermore, lifestyle inflammation scores (LIS) have been introduced to address lifestyle characteristics' cumulative contributions, including body mass index, physical activity, alcohol consumption, and smoking to body inflammation status $[13,14]$. Evidence about EDIP is still limited. Recently, two studies have suggested a positive link between EDIP and diabetes [15, 16]. Furthermore, the relationship between this index and other outcomes has been somewhat inconsistent [17-20]. To our knowledge, no study has yet investigated the relationship between DIS, LIS with risk of T2DM. Some previous studies have been assessed the association of these two novel inflammation scores with the risk of chronic diseases such as metabolic syndrome, cancers, inflammatory bowel disease (IBD), and CVD risk factors [21-24].

The current study is aimed to assess the associations of DIS, LIS, and EDIP with the incidence of T2DM among adult participants in a population-based study, Tehran Lipid and Glucose Study (TLGS).

\section{Method}

\section{Study participants}

The present study was conducted within the framework of the Tehran Lipid and Glucose Study (TLGS), which was conducted to determine the risk factors for noncommunicable diseases among a representative urban population of Tehran, including 15,005 participants aged $\geq 3$ years. The TLGS is an ongoing population-based prospective study initiated in 1999 (First phase), and its data are being collected prospectively at 3 y intervals. Written informed consent was obtained from all participants. All methods were carried out in accordance with relevant guidelines and regulations. Details of the TLGS have been reported previously [25].

In the fourth phase of the TLGS (2009-2011), 12,823 participants, 7956 randomly selected, agreed to complete the dietary assessment. For the current study, a total of 6560 individuals, aged $>20$ years old, with complete dietary data in the fourth examination of TLGS, as a baseline examination, were enrolled. Participants with underreporting or over-reporting dietary intakes (energy intakes of less than $800 \mathrm{kcal} / \mathrm{d}$ or more than $4500 \mathrm{kcal} / \mathrm{d}$, respectively; these values between the $\pm 3 \mathrm{SD}$ of mean energy intake in the study population), $(n=459)$, those on diabetes control diet $(n=205)$, participants with diabetes $(n=552)$, those with a history of myocardial infarction, cerebral vascular accident, cancers $(n=60)$, individuals with body mass index $(\mathrm{BMI})<18.5$ or $>40$ $\mathrm{kg} / \mathrm{m}^{2} \quad(n=207)$, lactating and pregnant women $(n=$ $116)$, and those with missing data of smoking $(n=9)$ were excluded in the baseline of the study. Some individuals fell into more than one exclusion category. From 5139 participants who were followed up until the sixth phase of TLGS (2015-18), over a mean period of 5.71 years, 515 participants were missed to follow up, and 4624 subjects remained for the final analyses (follow up rate $=90 \%)($ Fig. 1$)$.

\section{Dietary intake assessment}

Dietary intakes at baseline were assessed using a valid and reliable semi-quantitative food frequency 


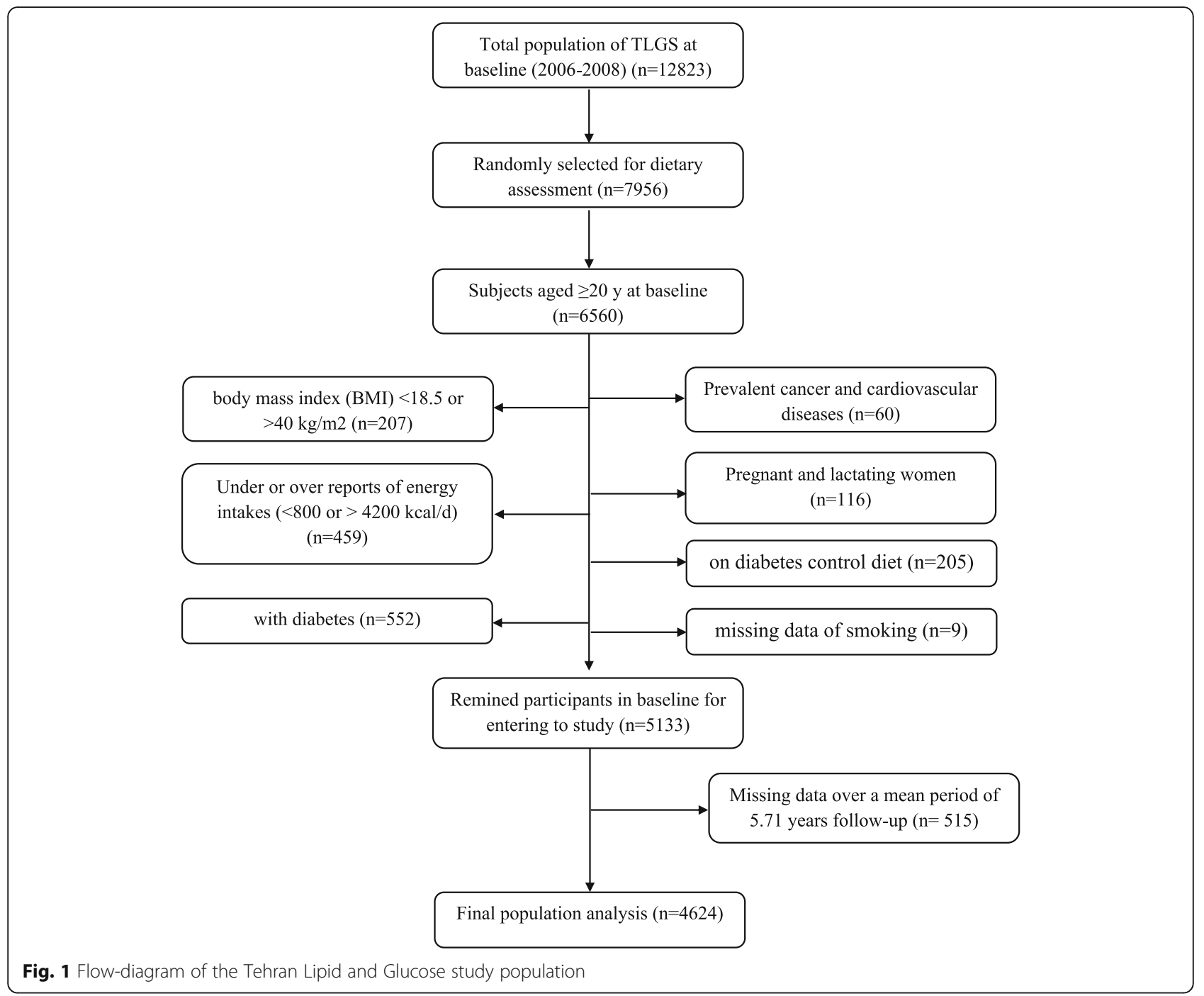

questionnaire (FFQ) [26]. The reliability and validity of the FFQ have been previously reported. During the previous year, the consumption frequency of each food item daily, weekly, or monthly was collected during a face-toface interview by trained and experienced dieticians. Portion sizes of consumed foods reported in household measures were then converted into grams. Using the United States Department of Agriculture (USDA) food composition table (FCT), energy and nutrient contents were computed. The Iranian FCT was used for local food items that were not available in USDA FCT.

\section{Physical activity assessment}

The modifiable activity questionnaire (MAQ) was used for assessing physical activity levels in participants. This questionnaire has previously been modified and validated among the Iranian population [27]; individuals were asked to report and identify the frequency and time spent on activities of light, moderate, hard, and very hard intensity, during the past 12 months, according to a list of everyday activities of daily life; physical activity levels were expressed as metabolic equivalent hours per week.

\section{Clinical and biochemical measurements}

Information on age, sex, medical history, medication use, and participants' smoking habits were collected by trained interviewers using pretested questionnaires. The subjects' body weight was measured to the nearest $100 \mathrm{~g}$ using digital scales (model 707, Seca, Hamburg, Germany) while lightly clothed and not wearing shoes. Height was measured to the nearest $0.5 \mathrm{~cm}$ with a tape measure (model 208 Portable Body Meter Measuring Device; Seca) while the subjects were barefoot in the standing position. Body mass index (BMI) was computed as weight in kilograms, divided by height in meters squared. Waist circumference (WC) was measured to the nearest $0.1 \mathrm{~cm}$ using an un-stretched tape meter, at 
the umbilicus level, over light clothing, without any pressure on the body surface.

BP was measured twice with a minimum interval of $30 \mathrm{~s}$ from the right arm after resting for at least $15 \mathrm{~min}$ while sitting on a chair, using a mercury sphygmomanometer and the Korotkoff sound technique with an accuracy of $2 \mathrm{mmHg}$; the mean of the two measurements was regarded as the participant's blood pressure.

Blood samples were taken and transferred into vacutainer tubes between 7:00 and 9:00 a.m., after a 12-14-h overnight fast, while subjects were in a sitting position. Blood samples were centrifuged within 30 to $45 \mathrm{~min}$ of collection. All biochemical analyses were performed using a Selectra 2 auto-analyzer at the TLGS research laboratory on the day of blood collection. Fasting blood sugar (FBS) was measured using an enzymatic colorimetric method with glucose oxidase. Inter- and intraassay CVs were both $2.2 \%$ for FBS [25]. Triglyceride (TGs) levels were measured using the enzymatic colorimetric method with glycerol phosphate oxidase. Interand intra-assay CVs for TGs were 0.6 and $1.6 \%$, respectively. Serum high-density lipoprotein-cholesterol (HDLC) was measured after precipitation of the apolipoprotein B-containing lipoproteins with phosphotungstic acid. Enzymatic colorimetric tests were used to assay total cholesterol (TC) with cholesterol esterase and cholesterol oxidase. Inter- and intra-assay CVs for both TC and HDL-C were 0.5 and $2 \%$, respectively. Friedewald formula was used to calculate low-density lipoproteincholesterol (LDL-C) expressed in $\mathrm{mg} / \mathrm{dL}$. Biochemical measurements were performed using commercial kits (Pars Azmoon Inc., Tehran, Iran).

\section{Calculation of indices}

Dietary data derived from FFQ were used to calculate inflammatory scores. The dietary (DIS) and lifestyle (LIS) inflammation scores were recently proposed by Byrd D.A et al. [13]. DIS includes 19 component initially, but because we have no information about supplement intake, we calculated the overall score with 18 food groups, including leafy greens and cruciferous vegetables, tomatoes, apples and berries, deep yellow or orange vegetables and fruit, other fruits, and real fruit juices, other vegetables, legumes, fish, poultry, red and organ meats, processed meats, added sugars, high-fat dairy, low-fat dairy and tea, nuts, other fats, refined grains, and starchy vegetables. We standardized each food group (to a mean of zero and SD of 1), and then the values were summed [13] (Table S1).

Physical activity, BMI, and smoking status were used to calculate the LIS score. Due to religious and legal restrictions in the Iranian population, alcohol is not consumed, or its consumption is not reported, so we ignored alcohol consumption to calculate LIS. First, a dummy variable was created from each component and then multiplied for proposed regression coefficients as follows [13] (Table S1).

Physical activity was categorized into tertiles, and participants in the first, second, and third tertiles give the score of $0.0,-0.18$, and -0.41 , respectively. Participants were categorized into average weight $(\mathrm{BMI}<25)$, overweight $(25 \leq \mathrm{BMI}<30)$, and obese $(\mathrm{BMI} \geq 30)$; and then respectively received 0.0. 0.89 and 1.57 scores. Also, the proposed regression coefficients for smokers vs. non-smokers were 0.50 vs. 0.0 , which were assigned. Finally, all the weighted values were summed to calculate the LIS score.

The EDIP scores were calculated based on the Tabung et al. study [14]. Since alcoholic drinks such as wine and beer are not common or maybe unreported in the Iranian population due to religious considerations, we do not include them in calculating indices. Because we have no food items as low-energy beverages in our FFQ, the food item was also excluded. Therefore we calculated EDIP score with 15 instead of 18 food group based on dietary intakes of processed meat (sausage), red meat (beef, or lamb), organ meat (beef, calf, or chicken liver), other fish (canned tuna, or fish), other vegetables (mixed vegetables, green pepper, cooked mushroom, eggplant, zucchini, or cucumber), refined grains (white bread, biscuit, white rice, pasta, or vermicelli), high-energy and low energy beverages (cola with sugar, carbonated beverages with sugar, fruit punch drinks), and tomatoes as pro-inflammatory group and tea, coffee, dark yellow vegetables (carrots, or squash), leafy green vegetables (cabbage, spinach, or lettuce), snacks (cracker, or potato chips), fruit juice (apple juice, cantaloupe juice, orange juice, or other fruit juice), pizza as an anti-inflammatory group. The mean daily intakes of each food group were multiplied by the proposed regression coefficients, and then all the weighted values were summed [14] (Table S1). Finally, the summed scores were divided by 1000 to reduce the magnitude of the scores. In every three indexes, a higher score indicates a more pro-inflammatory diet and vice versa.

\section{Definitions of terms}

T2DM was defined based on the American Diabetes Association's criteria as having FBS levels $\geq 126 \mathrm{mg} / \mathrm{dl}$ or 2 $\mathrm{h}$ post- $75 \mathrm{~g}$ glucose loads $\geq 200 \mathrm{mg} / \mathrm{dl}$ or treating with hypoglycaemic drugs [28]. Also, for the definition of hypertension, subjects who had systolic blood pressure levels higher than 140 or diastolic blood pressure levels of $90 \mathrm{mmHg}$ or consumed antihypertensive medications were considered hypertensive [29].

\section{Statistical analysis}

Data analyses were conducted using the Statistical Package for Social Sciences (version 20.0; SPSS Inc., Chicago, IL). The normality of variables was assessed using 
histogram charts and Kolmogorov-Smirnov analysis. Baseline characteristics of subjects were expressed as mean \pm SD or median (25-75) interquartile range (IQR) for continuous variables and percentage for categorical variables, respectively. An independent sample t-test and chi-square were used for comparing the quantitative and qualitative variables between diabetic and healthy participants.

Individuals' duration of follow-up (in the year) were calculated from baseline to the time at which an event (definitive diagnosis of diabetes by endocrinologist based on the criteria as mentioned above) occurred for the first time (event date) or the last date of follow up examination, whichever occurred first. The event date of occurrence of the diabetes was determined as mid-time between the date of the follow-up visit at which the diabetes was detected for the first time and the most recent follow-up visit preceding the diagnosis.

Subjects were categorized according to quartiles of EDIP, DIS, and LIS. Cox proportional hazard regression used to estimate the hazard ratio and $95 \%$ confidence intervals (HRs and 95\% CIs) of diabetes incident across quartiles of the EDIP, DIS, and LIS scores adjusted for potential confounders including age, sex, BMI, physical activity, smoking, daily energy intake, education level, hypertension, fasting blood sugar, and TG to HDL ratio.

We conducted a sensitivity analysis by excluding the smokers, participants with a family history of diabetes, and hypertensive patients; then, we repeated the analysis of the association between inflammatory factors with diabetes incidents. As smoking was a component of the LIS score, by excluding the smokers, LIS was unpredictable, so sensitivity analysis among the nonsmoker's population was just conducted for assessing the association of EDIP and DIS with diabetes incidence.

We also stratified our data based on the BMI (BMI < $25, \mathrm{BMI} \geq 25$ ) and physical activity categories (lower than the median, higher than the median) and analyzed the association between EDIP and DIS with diabetes incidents adjusted for the variables as mentioned above across these categories. $P$-values $<.05$ were considered as statistically significant.

\section{Result}

The mean \pm SD for the age and BMI of the study population were $40.8 \pm 12.7$ years and $27.1 \pm 4.1 \mathrm{Kg} . \mathrm{m} 2$, respectively, and $45.1 \%$ of participants were male. Also, the median (IQR) of EDIP, LIS, and DIS were $0.47(0.30$, $0.70), 0.71(0.00,1.16)$, and $0.06(-0.51,0.61)$, respectively. During an average of 5.71 years of follow-up, 329 (7.1\%) new cases of diabetes were ascertained.

The Baseline characteristics and dietary intakes of patients are presented in Table 1. Compared with nondiabetic subjects, participants with diabetes were significantly older and had higher BMI, FBS, TGs, SBP, DBP, and TG: HDL ratio, a higher percentage of hypertension, and lower HDL-C academic education level.

The components of three inflammatory indices, including EDIP, LIS, and DIS among diabetic and healthy participants, are shown in Table 2. The intakes of energy, macronutrients were not significantly different between the two groups. In comparison to non-diabetic patients, diabetic subjects had a healthier diet based on the DIS because they have a significantly lower score of

Table 1 Baseline characteristics of participants according to the development of diabetes during the follow-up

\begin{tabular}{llll}
\hline & Diabetes $(\boldsymbol{n}=\mathbf{3 2 9})$ & Healthy $(\boldsymbol{n}=\mathbf{4 2 9 5})$ & $\boldsymbol{P}$-value \\
\hline Age(year) & $47.7(12.4)$ & $40.3(12.6)$ & $<0.001$ \\
Male, $\mathrm{n}(\%)$ & $158(48.0)$ & $1928(44.9)$ & 0.271 \\
Body mass index $\left(\mathrm{Kg} / \mathrm{m}^{2}\right)$ & $29.7(4.1)$ & $27.0(4.1)$ & $<0.001$ \\
Physical Activity (Met.min/wk) & $73.3(53.6)$ & $74.5(54.2)$ & 0.356 \\
Academic education (graduated), $\mathrm{n}(\%)$ & $65(19.8)$ & $1297(30.2)$ & $<0.001$ \\
Employed, $\mathrm{n}(\%)$ & $272(82.7)$ & $3611(84.1)$ & 0.411 \\
Smoking, $\mathrm{n}(\%)$ & $40(12.2)$ & $482(11.2)$ & 0.609 \\
Biochemical data & & $92.0(7.8)$ & $<0.001$ \\
Fasting blood sugar (mg/dl) & $101.9(11.1)$ & $133.2(80.6)$ & $<0.001$ \\
Triglycerides (mg/dl) & $189.1(95.6)$ & $47.8(11.4)$ & $<0.001$ \\
High density lipoprotein- Cholesterol (mg/dl) & $44.4(11.0)$ & $3.1(2.6)$ & $<0.001$ \\
TG:HDL ratio & $4.7(3.1)$ & $111.8(14.8)$ & $<0.001$ \\
Systolic blood pressure (mmHg) & $122.1(17.9)$ & $75.2(10.3)$ & $<0.001$ \\
Diastolic blood pressure (mmHg) & $79.6(12.2)$ & $583(13.6)$ & $<0.001$ \\
Hypertension (\%) & $107(32.5)$ &
\end{tabular}

Data are presented as mean (SD) for continuous variable and number (percent) for categorical variables TG:HDL ratio Triglycerides: High density lipoprotein- Cholesterol ratio 
Table 2 Dietary EDIP, DIS and LIS components intakes of participants' according to their status of type 2 diabetes: Tehran Lipid and Glucose study

\begin{tabular}{|c|c|c|c|}
\hline & Diabetes $(n=329)$ & Healthy $(n=4295)$ & $P$-value \\
\hline \multicolumn{4}{|l|}{ Nutrients } \\
\hline Energy (Kcal/d) & $2407(704)$ & $2428(747)$ & 0.593 \\
\hline Carbohydrate (\% of energy) & $58.9(7.1)$ & $59.0(8.5)$ & 0.845 \\
\hline Protein (\% of energy) & $15.0(2.6)$ & $15.0(6.9)$ & 0.949 \\
\hline Fat (\% of energy) & $29.6(6.2)$ & $30.0(14.6)$ & 0.310 \\
\hline \multicolumn{4}{|l|}{ EDIP component } \\
\hline EDIP score & $0.59(0.44)$ & $0.53(0.39)$ & 0.032 \\
\hline Processed meat(serving/d) & $0.02(0.00-0.04)$ & $0.02(0.00-0.04)$ & 0.611 \\
\hline Red meat(serving/d) & $0.09(0.06-0.16)$ & $0.09(0.05-0.16)$ & 0.419 \\
\hline Organ meat(serving/d) & $0.01(0.00-0.02)$ & $0.01(0.00-0.02)$ & 0.464 \\
\hline Other fish(serving/d) & $0.06(0.03-0.13)$ & $0.06(0.03-0.13)$ & 0.841 \\
\hline Other vegetables(serving/d) & $2.67(2.28)$ & $2.38(1.91)$ & 0.024 \\
\hline Refined grains(serving/d) & $3.52(2.92)$ & $3.34(2.49)$ & 0.303 \\
\hline High-energy beverages(serving/d) & $0.05(0.01-0.13)$ & $0.05(0.01-0.11)$ & 0.713 \\
\hline Tomatoes(serving/d) & $0.64(0.32-1.12)$ & $0.64(0.32-1.12)$ & 0.138 \\
\hline Tea(serving/d) & $2.56(2.09)$ & $2.43(2.07)$ & 0.241 \\
\hline Coffee(serving/d) & $0.00(0.00-0.02)$ & $0.00(0.00-0.02)$ & 0.998 \\
\hline Dark yellow vegetables(serving/d) & $0.13(0.05-0.29)$ & $0.13(0.05-0.29)$ & 0.609 \\
\hline Leafy green vegetables(serving/d) & $0.43(0.21-0.77)$ & $0.37(0.18-0.68)$ & 0.174 \\
\hline Snacks(serving/d) & $0.13(0.01-0.36)$ & $0.15(0.03-0.41)$ & 0.019 \\
\hline Fruit juice(serving/d) & $0.04(0.01-0.11)$ & $0.04(0.01-0.12)$ & 0.771 \\
\hline Pizza(serving/d) & $0.01(0.00-0.03)$ & $0.01(0.00-0.03)$ & 0.852 \\
\hline \multicolumn{4}{|l|}{ DIS component } \\
\hline DIS score & $-0.07(-3.36,2.89)$ & $0.07(-0.50-0.62)$ & 0.006 \\
\hline Leafy greens and Cruciferous vegetables (g/d) & $23.4(11.1-41.6)$ & $20.7(10.2-37.5)$ & 0.263 \\
\hline Tomatoes(g/d) & $81.0(45.6-173.8)$ & $79.0(41.8-137.8)$ & 0.157 \\
\hline Apples and berries(g/d) & $68.8(35.7-131.3)$ & $64.2(27.3-126.7)$ & 0.461 \\
\hline Deep yellow or orange Vegetables and fruit(g/d) & $40.5(20.0-79.9)$ & $37.2(19.4-74.1)$ & 0.786 \\
\hline Other fruits and real fruit juices(g/d) & $233(128-390)$ & $222(116-383)$ & 0.333 \\
\hline Other vegetables $(\mathrm{g} / \mathrm{d})$ & $187(128)$ & $163(108)$ & 0.001 \\
\hline Legumes $(\mathrm{g} / \mathrm{d})$ & $47.9(43.8)$ & $46.2(42.2)$ & 0.503 \\
\hline Fish(g/d) & $6.4(3.3-14.5)$ & $6.7(3.6-14.6)$ & 0.841 \\
\hline Poultry(g/d) & $24.3(12.1-36.4)$ & $24.3(12.1-36.4)$ & 0.318 \\
\hline Red and organ meats(g/d) & $33.9(23.4)$ & $36.1(27.1)$ & 0.103 \\
\hline Processed meats $(\mathrm{g} / \mathrm{d})$ & $2.66(0.49-5.33)$ & $2.41(0.49-5.33)$ & 0.611 \\
\hline Added sugars(g/d) & $57.0(28.8-120.9)$ & $59.8(31.4-117.1)$ & 0.920 \\
\hline High-fat dairy(g/d) & $82.9(20.4-234.6)$ & $99.9(30.3-233.9)$ & 0.418 \\
\hline Low-fat dairy(g/d) & $193(166)$ & $181(151)$ & 0.209 \\
\hline Coffee and tea(g/d) & $628(507)$ & $595(499)$ & 0.246 \\
\hline $\operatorname{Nuts}(g / d)$ & $4.59(2.07-10.41)$ & $4.40(2.06-9.11)$ & 0.133 \\
\hline Other fats(g/d) & $24.5(18.8)$ & $25.7(19.4)$ & 0.274 \\
\hline Refined grains and Starchy vegetables(g/d) & $477(205)$ & $494(218)$ & 0.151 \\
\hline
\end{tabular}

\section{LIS Component}


Table 2 Dietary EDIP, DIS and LIS components intakes of participants' according to their status of type 2 diabetes: Tehran Lipid and Glucose study (Continued)

\begin{tabular}{llll}
\hline & Diabetes $(\boldsymbol{n}=\mathbf{3 2 9})$ & Healthy $(\boldsymbol{n}=\mathbf{4 2 9 5})$ & $\boldsymbol{P}$-value \\
\hline LIS score & $0.98(0.71-1.39)$ & $0.71(0.00-0.98)$ & $<.001$ \\
Current smoker & $40(12.2)$ & $428(11.2)$ & 0.609 \\
Physical activity categories & & & 0.787 \\
$\quad$ Moderately physically active & $108(32.8)$ & $1428(33.2)$ \\
$\quad$ Heavily physically active & $108(32.8)$ & $1428(33.2)$ \\
BMI categories & $138(41.9)$ & $1928(44.9)$ \\
$\quad$ Overweight (BMI =25-29.9) & $150(45.6)$ & $937(21.8)$ \\
$\quad$ Obese (BMI $\geq 30)$ & &
\end{tabular}

Data are presented as mean (standard deviation) for normally distributed variables and median (25-75 interquartile range) for skewed variables EDIP empirical dietary inflammatory pattern, DIS dietary inflammation scores, LIS lifestyle inflammation scores

DIS and also higher "other vegetables" consumption as a DIS component $(P$-value $=0.05)$. Compared with nondiabetic subjects, participants with diabetes had higher EDIP scores, and among EDIP components, "other vegetables" consumption, however, had a lower intake of snacks $(P$-value $=0.05)$. LIS scores and percentage of obesity or overweight (higher BMI) as a component of LIS were higher among diabetic patients $(P$-value $=0.05)$.

The association of EDIP, DIS, and LIS with the risk of incident diabetes is shown in Table 3. In the age and sex-adjusted model, the odds of diabetes were higher in individuals in the highest quartiles of the EDIP $(\mathrm{HR}=$

Table 3 Hazard ratio (95\% confidence intervals) of developing diabetes based on quartiles of inflammatory indices

\begin{tabular}{|c|c|c|c|c|c|}
\hline & \multicolumn{4}{|c|}{ Quartiles of scores } & \multirow[t]{2}{*}{$P$ for trend } \\
\hline & $\overline{\mathrm{Q} 1}$ & Q2 & Q3 & Q4 & \\
\hline \multicolumn{6}{|l|}{ EDIP } \\
\hline Median score & 0.19 & 0.39 & 0.57 & 0.91 & \\
\hline Case/Total & $71 / 1156$ & $90 / 1154$ & $74 / 1156$ & $94 / 1155$ & \\
\hline Follow-up time & $5.76(1.51)$ & $5.69(1.55)$ & $5.74(1.57)$ & $5.66(1.640$ & \\
\hline Model $1^{*}$ & 1.00 (Ref) & $1.36(0.99-1.88)$ & $1.16(0.83-1.63)$ & $1.60(1.17-2.19)$ & 0.009 \\
\hline Model $2^{\dagger}$ & 1.00 (Ref) & $1.36(0.99-1.88)$ & $1.11(0.79-1.58)$ & $1.55(1.11-2.17)$ & 0.026 \\
\hline Model $3^{\ddagger}$ & 1.00 (Ref) & $1.40(1.01-1.93)$ & $1.32(0.86-1.72)$ & $1.52(1.08-2.14)$ & 0.038 \\
\hline \multicolumn{6}{|l|}{ DIS } \\
\hline Follow-up time & $5.71(1.58)$ & $5.71(1.57)$ & $5.69(1.58)$ & $5.74(1.55)$ & \\
\hline Median score & -1.01 & -0.21 & 0.32 & 1.01 & \\
\hline Case/Total & $100 / 1155$ & $87 / 1155$ & $80 / 1155$ & $62 / 1155$ & \\
\hline Model $1^{*}$ & 1.00 (Ref) & $0.96(0.72-1.30)$ & $0.95(0.70-1.28)$ & $0.83(0.59-1.15)$ & 0.286 \\
\hline Model $2^{\dagger}$ & 1.00 (Ref) & $1.06(0.79-1.44)$ & $1.02(0.75-1.40)$ & $0.91(0.65-1.28)$ & 0.640 \\
\hline Model $3^{\ddagger}$ & 1.00 (Ref) & $0.85(0.72-1.31)$ & $0.89(0.71-1.34)$ & $0.36(0.61-1.20)$ & 0.418 \\
\hline \multicolumn{6}{|l|}{ LIS } \\
\hline Follow-up time & $5.80(1.48)$ & $5.80(1.51)$ & $5.73(1.54)$ & $5.51(1.72)$ & \\
\hline Median score & -0.18 & 0.48 & 0.89 & 1.39 & \\
\hline Case/Total & 34 / 1297 & $84 / 1401$ & $53 / 721$ & $157 / 1201$ & \\
\hline Model $1^{*}$ & 1.00 (Ref) & $1.99(1.31-3.01)$ & $2.29(1.46-3.59)$ & $4.26(2.88-6.30)$ & $<0.001$ \\
\hline Model $2^{n}$ & 1.00 (Ref) & 1.97 (1.30-2.99) & $2.26(1.44-3.56)$ & $4.18(2.83-6.20)$ & $<0.001$ \\
\hline Model $3^{\S}$ & 1.00 (Ref) & $1.53(1.01-2.34)$ & $1.38(0.87-2.21)$ & $2.41(1.61-3.60)$ & $<0.001$ \\
\hline
\end{tabular}

*Model 1: adjusted for age and sex

${ }^{\dagger}$ Model 2: adjusted for model 1 and energy, body mass index, smoking, physical activity, education level

${ }^{\ddagger}$ Model 3: adjusted for model 2 and hypertension, fasting blood sugar, and TG to HDL ratio

"Model2: adjusted for model 1 and energy and education level

${ }^{\S}$ Model 3: adjusted for model 1 and energy, education level, hypertension, fasting blood sugar, and TG to HDL ratio 
1.60; 95\%CI:1.17-2.19, $\mathrm{p}$ for trend $=0.009)$ and LIS $(\mathrm{HR}=$ 4.26; 95\%CI:2.88-6.30, $\mathrm{p}$ for trend $<0.001$ ) compared to those in the lowest quartile of these scores; However, there was no statically significant association between DIS and risk of diabetes ( $\mathrm{HR}=0.83$; 95\%CI:0.59-1.15, $\mathrm{p}$ for trend $=0.286$ ). In the final model (model 3 ), after further adjustment for BMI, smoking, physical activity (only for EDIP and DIS), energy, education level, hypertension, fasting blood sugar, and TG to HDL ratio, participants in the highest vs. lowest quartile of LIS (HR $=2.41$; 95\%CI:1.613.60, P for trend $<0.001)$, and EDIP (HR $=1.52$; 95\%CI:0. $1.08-2.14$, $\mathrm{P}$ for trend $=0.0 .038$ ) has a higher risk of diabetes incident. However, according to each of the three models, a higher score of DIS showed no significant association with the risk of diabetes.

Table 4 presents the association of inflammatory indices and the risk of diabetes incident among the participants who were on the highest compared with lowest quartiles of EDIP, DIS, and LIS using a sensitivity analysis based on the excluding of smokers, participants with family history of diabetes, and hypertensive patients.

In the final adjusted models for potential variables, the highest vs. lowest quartile of EDIP significantly associated with a higher risk of T2DM (HR: 1.65, 95\% CI 1.15-2.38, P for trend:0.018), (HR: 1.55 , 95\% CI $1.07-$ 2.25, P for trend:0.064), (HR: 1.59, 95\% CI 1.05-2.40, P for trend:0.049) after excluding of smokers, participants with family history of diabetes, and hypertensive patients, respectively. However, no significant association was observed between a higher score of DIS and the risk of T2DM.

Also, the direct association between LIS and diabetes incident was repeated in sensitivity analysis; as the HR and $95 \% \mathrm{CI}$ of diabetes incidents among participants in the highest compared to lowest quartiles of LIS were 2.20 (1.44-3.36), P for trend $<0.001$ and 2.41 (1.51$3.83)$, $\mathrm{P}$ for trend $<0.001$ after excluding the participants with family history of diabetes and hypertensive patients, respectively.

Stratified analyses were performed based on the BMI $(\mathrm{BMI}<25, \mathrm{BMI} \geq 25)$ and physical activity classification (lower than the median, higher than the median) to assess the association of DIS and EDIP with risk of diabetes incidents across these categories, and findings were reported in Fig. 2. Based on the stratified analysis, in participants with a $\mathrm{BMI} \geq$ of 25 , after adjusting for variables as mentioned earlier, a higher score of EDIP significantly associated with a higher risk of T2DM (HR: 1.46, 95\% CI 1.02-2.08, P for trend:0.070) (Fig. 2A). However, no significant association was observed between a higher score of EDIP and the risk of T2DM in subjects with $\mathrm{BMI}<25$. Also, there is no significant association between the higher score of DIS risk of T2DM in participants based on BMI classification $(\mathrm{BMI}<25$, $\mathrm{BMI} \geq 25$ ) (Fig. 2A). Furthermore, stratified analysis according to physical activity categories (lower than the median, higher than the median) showed no significant relationship between DIS and EDIP score with risk of T2DM (Fig. 2B).

\section{Discussion}

In the present study, we used three indices, including EDIP, DIS, and LIS, to evaluate the association between the inflammatory potential of diet and lifestyle with the risk of diabetes among Tehranian adults. Our findings suggest that greater adherence to a pro-inflammatory diet with higher scores of EDIP associated with a higher

Table 4 Hazard ratio (95\% confidence intervals) of developing diabetes based on quartiles of inflammatory indices after excluding the smokers, participants with family history of diabetes, and Hypertensive patients

\begin{tabular}{|c|c|c|c|c|c|}
\hline & \multicolumn{4}{|c|}{ Quartiles of scores } & \multirow[t]{2}{*}{ P for trend } \\
\hline & Q1 & Q2 & Q3 & Q4 & \\
\hline \multicolumn{6}{|c|}{ Smoker excluded $(N=4102)$} \\
\hline EDIP & 1.00 (ref) & $1.47(1.03-2.08)$ & $1.30(0.89-1.88)$ & $1.65(1.15-2.38)$ & 0.018 \\
\hline DIS & 1.00 (ref) & $1.02(0.74-1.41)$ & $1.00(0.72-1.40)$ & $0.88(0.61-1.26)$ & 0.522 \\
\hline \multicolumn{6}{|c|}{ participants with family history of diabetes excluded $(N=4152)^{\ddagger}$} \\
\hline EDIP & 1.00 (ref) & $1.56(1.10-2.21)$ & $1.30(0.90-1.89)$ & $1.55(1.07-2.25)$ & 0.064 \\
\hline DIS & 1.00 (ref) & $1.00(0.72-1.39)$ & $0.99(0.71-1.39)$ & $0.93(0.65-1.33)$ & 0.717 \\
\hline LIS & 1.00 (ref) & $1.50(0.97-2.31)$ & $1.32(0.80-2.16)$ & $2.20(1.44-3.36)$ & $<0.001$ \\
\hline \multicolumn{6}{|c|}{ Hypertensive patients excluded $(N=3926)^{\S}$} \\
\hline EDIP & 1.00 (ref) & $1.40(0.94-2.07)$ & $1.31(0.86-2.00)$ & $1.59(1.05-2.40)$ & 0.049 \\
\hline DIS & 1.00 (ref) & $1.01(0.71-1.46)$ & $1.11(0.76-1.61)$ & $0.80(0.53-1.21)$ & 0.448 \\
\hline LIS & 1.00 (ref) & $1.58(0.98-2.54)$ & $1.65(0.98-2.78)$ & $2.41(1.51-3.83)$ & $<0.001$ \\
\hline
\end{tabular}

Fadjusted for age, sex, energy, body mass index, smoking, physical activity, education level, hypertension, fasting blood sugar, and TG to HDL ratio

sadjusted for age, sex, energy, education level, hypertension, fasting blood sugar, and TG to HDL ratio 


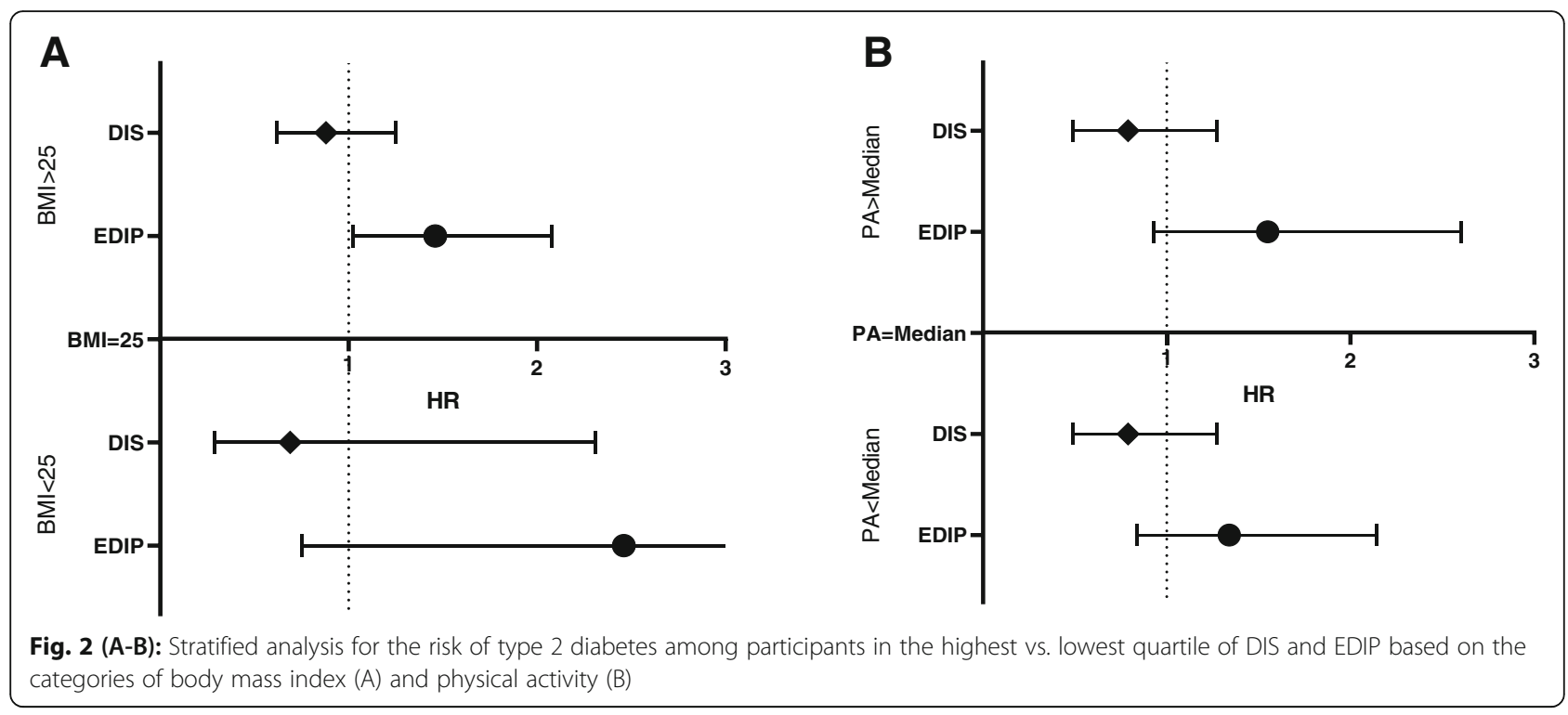

risk of diabetes, while no significant association was found between DIS score and the risk of diabetes; also, we found a positive association between LIS score, as an indicator of lifestyle influences on inflammatory status, and incidence of diabetes after 5.71 years follow-up. Also, sensitivity analyses based on BMI and physical activity classification somewhat showed repeated results on the association of DIS and EDIP with risk of T2DM. However, stratified analysis based on BMI categorization suggested that a higher score of EDIP represents a proinflammatory dietary pattern, increases the risk of developing T2DM if individuals have an excessive BMI.

To the best of our knowledge, the association between DIS and LIS and the risk of diabetes has not been investigated previously. However, recently two studies have been conducted on the relationship of EDIP with diabetes risk $[15,16]$. Also, other epidemiological studies have assessed the association between these inflammatory indices and the risk of various chronic diseases such as metabolic syndrome, CVD risk factors, cancers, and IBD, where the reported findings are controversial [19, 20, 30-32]. Shakeri, $\mathrm{Z}$ et al. investigated the relationship between EDIP and MetS and its component and found that a pro-inflammatory diet is a risk factor for the development of MetS, hyperglycemia, low HDL-C, and central obesity [19]. Also, Soltani et al., In a crosssectional study, observed that overweight/obese individuals with higher EDIP scores have an increased odds of unhealthy metabolic phenotype, high FBS, low-HDL-C, and lower waist circumference [20]. These two crosssectional studies showed that EDIP is associated with greater odds of diabetes risk factors. Two prospective studies conducted by Byrd et al. found that the inflammatory potential of diet, measured by DIS and LIS [33,
34], may increase the risk of all-cause, cancer- and CVD-specific mortality [33] and colorectal cancer [34].

Furthermore, two prospective studies showed that a higher EDIP score could increase the risk of colorectal cancer [35]; however, other investigations on ovarian cancer [32] and bladder cancer [30] was found no association. Our findings on the association between EDIP and LIS score with the risk of diabetes incidence are consistent with the results of three cross-sectional studies that observed a higher DII score positively associated with a higher risk of diabetes [8-10]. Also, in a cohort study by Laouali et al., an adapted dietary inflammatory index (ADII) showed that greater adherence to an antiinflammatory diet is associated with a lower risk of T2DM [11].

Our findings showed a $56 \%$ higher risk of diabetes among participants in the highest quartile of EDIP than those in the lowest quartile. These results are in line with two recently published prospective studies $[15,16]$. Lee et al. demonstrated that individuals who consumed a pro-inflammatory diet (determined by higher EDIP score) are at three-time higher risk of diabetes [16]. Another study among postmenopausal women suggests that participants in the highest quintile of EDIP score have a $0.45 \%$ greater risk of diabetes; they also found that reducing the inflammatory potentials of the diet may have more preventive effects against diabetes than focusing only on glycemic foods [15]. EDIP, as a datadriven index, has focused mainly on that part of the diet, which seems to have more positive correlations with inflammatory markers, as we found positive values of EDIP for most participants (about 97\%), which shows this score highlights pro-inflammatory aspects of diet. The impact of the major anti-inflammatory contributors 
of the score was attenuated in our study for some reason; consumption of alcoholic beverages was unreported in our data due to religious considerations, and also coffee and pizza intake were too low. So, if these limitations did not exist, the individuals might be ranked in a broader range of scores, and it provides a better condition for comparing participants in different levels of EDIP.

In the present study, unlike the EDIP index, the DIS showed no association with the risk of T2DM. This finding mostly resulted from nutritional behavior differences between diabetic and non-diabetic participants as we observed a relatively healthier diet among diabetic participants. Besides, DIS is a dietary inflammatory index based on food groups that focus on a wide range of dietary foods; however, it provides a more realistic assessment of usual dietary intake. The interaction of anti/ pro-inflammatory foods can attenuate its estimation abilities for disease risk. Moreover, this index has been developed and validated for the US population, and the given weights have been calculated based on their dietary patterns and eating habits, so further studies are needed to assess its applicability in our population; as in our study, unexpectedly non-diabetic participants significantly had a higher DIS score. In addition, based on our findings, the LIS score was more robust than the DIS and EDIP in the estimation of the increment T2DM incident; This substantial increase in the risk of T2DM was seen especially in the fourth quartile of LIS; based on baseline findings, individuals in the fourth quartile of LIS had an unfavorable condition in terms of the lifestyle components, including physical activity level (36\% low active), obesity ( $90 \%$ obese) and smoking (19\% smoked). Each of these environmental factors can be as important as the dietary pattern in increasing inflammatory conditions and predisposing individuals to chronic diseases such as T2DM. This claim and hypothesis can be confirmed by results of stratified analysis on the association of EDIP with risk of T2DM, which showed that when an unfavorable environmental factor such as obesity is combined with an improper diet, the inflammatory condition can be exacerbated and predispose individuals to T2DM. Therefore, it was expected that the cooperative contributions of major lifestyle-related determinants such as BMI, physical activity, and smoking to inflammation, as a single inflammatory index (LIS), showed a stronger relationship with increased risk of T2DM than the DIS as an alone dietary inflammatory score.

LIS, which addresses cooperative contributions of main lifestyle-related factors, including BMI, physical activity, and smoking to inflammation, showed a more substantial relationship with diabetes than DIS and EDIP. It seems the combination of the LIS components at the continuous form with dietary pro/anti-inflammatory foods in one index can considerably improve the ability to estimate the risk of diabetes. Lifestyle-related factors such as BMI, physical activity, and smoking can significantly influence the inflammatory status and insulin homeostasis. High BMI and increased adipose tissue are positively associated with inflammatory markers such as TNF- $\alpha$, insulin resistance, and $\beta$-cell dysfunction [6, 10, 36-38]. Furthermore, smoking has been proposed as an independent risk factor for diabetes via its detrimental impact on $\beta$-cells dysfunction and IR, mostly related to excessive production of harmful tissues, up-regulating inflammatory biomarkers, and cytokines [39-41]. Also, cigarette nicotine exposure significantly decreases insulin sensitivity through $\mathrm{p} 44 / \mathrm{p} 42$ MAPK and mTOR pathways [40]. Moderate to high physical activity is another LIS component that has protective effects on chronic inflammation via its ability to improves plasma antioxidant capacity, inducing antiinflammatory cytokines production, reducing vascular wall inflammation [42, 43], desirable alteration in the lipid-deposition pattern and lowering body fat mass through negative energy balance [44].

This study had several strengths; it was a relatively large population-based prospective study with long-term follow-up, which firstly assessed the association of LIS and DIS with the risk of diabetes incidence globally and EDIP in an Asian population. We also used valid and reliable food-frequency and physical activity questionnaires for dietary and physical activity and filled them with expert interviewers during a face-to-face interview. Despite these strengths, some limitations of the present study should also be mentioned. Firstly, 15 out of 18 food groups were used to calculate the EDIP score in this study. Some items were also excluded in the calculation of DIS and LIS. The final scores were computed based on 18 instead of 19 for DIS and 3 instead of 4 for LIS. Secondly, similar to all nutritional studies using FFQ, measurement error is a potential concern. Thirdly, there is possible residual confounding that we cannot exclude due to unknown or unmeasured factors. Another possible limitation is that these indices have been validated for the US population, and using the given weight may not be favorably applicable in other populations. However, the lack of validated and reliable inflammatory biomarker panel and similar previous studies with a different population lead us to use these indices in our study.

\section{Conclusions}

This prospective, population-based study showed that higher EDIP and LIS scores were associated with an 
increased risk of diabetes in adults; however, DIS showed no significant association with diabetes incidence.

Further epidemiological studies are needed to address the role of the inflammatory potential of diet and lifestyle and their combinations in the risk of diabetes and its potential mechanisms.

\section{Abbreviations}

T2DM: Type 2 diabetes mellitus; CVD: Cardiovascular disease; EDIP: Empirical dietary inflammatory pattern; DIS: Dietary inflammation scores; LIS: Lifestyle inflammation scores; DII: Dietary inflammatory index; ADII: Adapted dietary inflammatory index; FBS: Fasting blood sugar; IR: Insulin resistance; CRP: Creactive protein; HDL-C: High-density lipoprotein-cholesterol; LDL-C: lowdensity lipoprotein-cholesterol; TG: Triglyceride; BP: Blood pressure; MAPK: Mitogen-activated protein kinase; mTOR: Mammalian target of rapamycin; USDA: United States department of agriculture; FCT: Food composition Table; BMl: Body mass index; WC: Waist circumference; FFQ: Food Frequency Questionnaire; Cl: Confidence interval; SD: Standard deviation; IQR: Interquartile range; OR: Odds ratio

\section{Supplementary Information}

The online version contains supplementary material available at https://doi. org/10.1186/s12889-021-11327-1.

Additional file 1: Table S1. Components of the EDIP, DIS, and LIS indices.

\section{Acknowledgments}

We express appreciation to the participants in the Tehran Lipid and Glucose Study for their enthusiastic support and the staff of the Research Institute for Endocrine Sciences, Tehran Lipid, and Glucose Study Unit, for their valuable help. We also acknowledge N. Shiva for critically editing the English grammar and syntax of the manuscript.

\section{Authors' contributions}

FT and HF conceptualized and designed the study. FT, NM, and HF drafted the initial manuscript; FT, MHS, and EM analyzed and interpreted the data; PM, NM, and FA supervised the project; all authors have read and approved the final version of the manuscript.

\section{Funding}

This work was funded by a grant from the Shahid Beheshti University of Medical Sciences, Tehran, Iran.

\section{Availability of data and materials}

The datasets analysed in the current study are available from the corresponding author on reasonable request.

\section{Declarations}

\section{Ethics approval and consent to participate}

Written informed consent was obtained from all participants. All methods were carried out in accordance with relevant guidelines and regulations. The study protocol was approved by the ethics research committee of the Research Institute for Endocrine Sciences, Shahid Beheshti University of Medical Sciences, Tehran, Iran (Reference number: IR.SBMU.ENDOCRINE.REC.1399.041)

\section{Consent for publication}

Not applicable.

\section{Competing interests}

The authors declared that there is no conflict of interest.

\section{Author details}

Tutrition and Endocrine Research Center, Research Institute for Endocrine Sciences, Shahid Beheshti University of Medical Sciences, Tehran, Iran. ${ }^{2}$ Department of Nutrition, School of Public Health, Iran University of Medical Sciences, Tehran, Iran. ${ }^{3}$ Endocrine Research Center, Research Institute for Endocrine Sciences, Shahid Beheshti University of Medical Sciences, Tehran, Iran

Received: 6 February 2021 Accepted: 18 June 2021

Published online: 02 July 2021

\section{References}

1. Werfalli M, Raubenheimer P, Engel M, Peer N, Kalula S, Kengne AP, et al. Effectiveness of community-based peer-led diabetes self-management programmes (COMP-DSMP) for improving clinical outcomes and quality of life of adults with diabetes in primary care settings in low and middleincome countries (LMIC): a systematic review and meta-analysis. BMJ Open. 2015;5(7):e007635. https://doi.org/10.1136/bmjopen-2015-007635.

2. Saeedi P, Petersohn I, Salpea P, Malanda B, Karuranga S, Unwin N, et al. Global and regional diabetes prevalence estimates for 2019 and projections for 2030 and 2045: Results from the international diabetes federation diabetes atlas, 9<sup>th</sup> edition. Diabetes Res Clin Pract. 2019:157.

3. Furman D, Campisi J, Verdin E, Carrera-Bastos P, Targ S, Franceschi C, et al. Chronic inflammation in the etiology of disease across the life span. Nat Med. 2019;25(12):1822-32. https://doi.org/10.1038/s41591-019-0675-0.

4. Ridker PM. High-sensitivity C-reactive protein: potential adjunct for global risk assessment in the primary prevention of cardiovascular disease. Circulation. 2001;103(13):1813-8. https://doi.org/10.1161/01.CIR.103.13.1813.

5. Dandona P, Aljada A, Bandyopadhyay A. Inflammation: the link between insulin resistance, obesity and diabetes. Trends Immunol. 2004;25(1):4-7. https://doi.org/10.1016/j.it.2003.10.013.

6. Calder PC, Ahluwalia N, Brouns F, Buetler T, Clement K, Cunningham K, et al Dietary factors and low-grade inflammation in relation to overweight and obesity. Br J Nutr. 2011;106(Suppl 3):S5-78. https://doi.org/10.1017/S0007114 511005460 .

7. Schulze MB, Hu FB. Primary prevention of diabetes: what can be done and how much can be prevented? Annu Rev Public Health. 2005;26(1):445-67. https://doi.org/10.1146/annurev.publhealth.26.021304.144532.

8. Denova-Gutiérrez E, Muñoz-Aguirre P, Shivappa N, Hébert JR, TolentinoMayo L, Batis C, et al. Dietary inflammatory index and type 2 diabetes mellitus in adults: the diabetes mellitus survey of Mexico City. Nutrients. 2018;10(4):385. https://doi.org/10.3390/nu10040385.

9. King $D E$, Xiang J. The dietary inflammatory index is associated with diabetes severity. J Am Board Fam Med. 2019:32(6):801-6. https://doi.org/10.3122/ja bfm.2019.06.190092

10. Mtintsilana A, Micklesfield LK, Chorell E, Olsson T, Shivappa N, Hebert JR, et al. Adiposity mediates the association between the dietary inflammatory index and markers of type 2 diabetes risk in middle-aged black south African women. Nutrients. 2019;11(6):1246. https://doi.org/10.3390/nu11 061246.

11. Laouali N, Mancini FR, Hajji-Louati M, El Fatouhi D, Balkau B, Boutron-Ruault $\mathrm{M}-\mathrm{C}$, et al. Dietary inflammatory index and type 2 diabetes risk in a prospective cohort of 70,991 women followed for 20 years: the mediating role of BMI. Diabetologia. 2019;62(12):2222-32. https://doi.org/10.1007/s0012 5-019-04972-0.

12. Shivappa N, Steck SE, Hurley TG, Hussey JR, Hebert JR. Designing and developing a literature-derived, population-based dietary inflammatory index. Public Health Nutr. 2014;17(8):1689-96. https://doi.org/10.1017/S13 68980013002115

13. Byrd DA, Judd SE, Flanders WD, Hartman TJ, Fedirko V, Bostick RM. Development and validation of novel dietary and lifestyle inflammation scores. J Nutr. 2019;149(12):2206-18. https://doi.org/10.1093/jn/nxz165.

14. Tabung FK, Smith-Warner SA, Chavarro JE, Wu K, Fuchs CS, Hu FB, et al. Development and validation of an empirical dietary inflammatory index. J Nutr. 2016;146(8):1560-70. https://doi.org/10.3945/jn.115.228718.

15. Jin Q, Shi N, Aroke D, Lee DH, Joseph JJ, Donneyong M, et al. Insulinemic and inflammatory dietary patterns show enhanced predictive potential for type 2 diabetes risk in postmenopausal women. Diabetes Care. 2021;44(3): 707-14. https://doi.org/10.2337/dc20-2216.

16. Lee DH, Li J, Li Y, Liu G, Wu K, Bhupathiraju S, et al. Dietary inflammatory and Insulinemic potential and risk of type 2 diabetes: results from three 
prospective US cohort studies. Diabetes Care. 2020;43(11):2675-83. https:// doi.org/10.2337/dc20-0815.

17. Mokhtary N, Mousavi SN, Sotoudeh G, Qorbani M, Kalantar Z, Koohdani FJOM. Association between dietary inflammatory indices (DII, EDII) and obesity with consideration of Insertion/Deletion Apo B polymorphism in type 2 diabetic patients. 2020;19:100241

18. Mirmiran P, Moslehi N, Morshedzadeh N, Shivappa N, Hébert JR, Farsi F, et al. Does the inflammatory potential of diet affect disease activity in patients with inflammatory bowel disease? Nutr J. 2019;18(1):65. https://doi. org/10.1186/s12937-019-0492-9.

19. Shakeri Z, Mirmiran P, Khalili-Moghadam S, Hosseini-Esfahani F, Ataie-Jafari A, Azizi F. Empirical dietary inflammatory pattern and risk of metabolic syndrome and its components: Tehran lipid and glucose study. Diabetol Metab Syndr. 2019;11(1):16. https://doi.org/10.1186/s13098-019-0411-4.

20. Soltani S, Moslehi N, Hosseini-Esfahani F, Vafa M. The association between empirical dietary inflammatory pattern and metabolic phenotypes in overweight/obese adults. Int J Endocrinol Metab. 2018;16(2):e60048. https:// doi.org/10.5812/ijem.60048

21. Li Z, Gao Y, Byrd DA, Gibbs DC, Prizment AE, Lazovich D, et al. Novel dietary and lifestyle inflammation scores directly associated with all-cause, allCancer, and all-cardiovascular disease mortality risks among women. J Nutr. 2021;151(4):930-9. https://doi.org/10.1093/jn/nxaa388.

22. Farhadnejad $H$, Parastouei $K$, Rostami $H$, Mirmiran P, Azizi FJD. Syndrome M. Dietary and lifestyle inflammatory scores are associated with increased risk of metabolic syndrome in Iranian adults. 2021;13(1):1-10.

23. Byrd DA, Judd SE, Flanders WD, Hartman TJ, Fedirko V, Agurs-Collins T, et al. Associations of Novel Dietary and Lifestyle Inflammation Scores With Incident Colorectal Cancer in the NIH-AARP Diet and Health Study. JNCl Cancer Spectrum. 2020;4(3).

24. Byrd DA, Judd S, Flanders WD, Hartman TJ, Fedirko V. Bostick RMJCE, et al. Associations of novel dietary and lifestyle inflammation scores with incident, sporadic colorectal adenoma. 2020;29(11):2300-8.

25. Azizi F, Ghanbarian A, Momenan AA, Hadaegh F, Mirmiran P, Hedayati M, et al. Prevention of non-communicable disease in a population in nutrition transition: Tehran lipid and glucose study phase II. Trials. 2009;10(1):5. https://doi.org/10.1186/1745-6215-10-5.

26. Esfahani FH, Asghari G, Mirmiran P, Azizi F. Reproducibility and relative validity of food group intake in a food frequency questionnaire developed for the Tehran lipid and glucose study. J Epidemiol. 2010;20(2):150-8. https://doi.org/10.2188/jea.JE20090083.

27. Momenan AA, Delshad M, Sarbazi N, Rezaei GN, Ghanbarian A, Azizi F. Reliability and validity of the modifiable activity questionnaire (MAQ) in an Iranian urban adult population. 2012.

28. Report of the expert committee on the diagnosis and classification of diabetes mellitus. Diabetes Care. 2003;26 Suppl 1:S5-20.

29. Mancia G, De Backer G, Dominiczak A, Cifkova R, Fagard R, Germano G, et al. 2007 guidelines for the Management of Arterial Hypertension: the task force for the Management of Arterial Hypertension of the European Society of Hypertension (ESH) and of the European Society of Cardiology (ESC). J Hypertens. 2007;25(6):1105-87. https://doi.org/10.1097/HJH.0b013e3281 fc975a.

30. Abufaraj M, Tabung FK, Shariat SF, Moschini M, Devore E, Papantoniou K, et al. Association between inflammatory potential of diet and bladder Cancer risk: results of 3 United States prospective cohort studies. J Urol. 2019;202(3):484-9. https://doi.org/10.1097/JU.00000000000000279.

31. Bridgman AC, Qureshi AA, Li T, Tabung FK, Cho E, Drucker AM. Inflammatory dietary pattern and incident psoriasis, psoriatic arthritis, and atopic dermatitis in women: a cohort study. J Am Acad Dermatol. 2019; 80(6):1682-90. https://doi.org/10.1016/j.jaad.2019.02.038.

32. Tabung FK, Huang T, Giovannucci EL, Smith-Warner SA, Tworoger SS, Poole EM. The inflammatory potential of diet and ovarian cancer risk: results from two prospective cohort studies. Br J Cancer. 2017;117(6):907-11. https://doi. org/10.1038/bjc.2017.246.

33. Byrd D, Holmes A, Judd S, Flanders W, Bostick R. Abstract 3287: Associations of whole food and lifestyle-based inflammation scores with all-cause, cancer- and cardiovascular disease-specific mortality. Cancer Res. 2017;77: 3287.

34. Byrd D, Bostick R, Judd S, Flanders W, Hartman T, Fedirko V, et al. Abstract 627: Associations of novel dietary and lifestyle inflammation scores with incident colorectal cancer in the National Institutes of Health-American Association of Retired Persons Diet and Health study. 2019. p. 627.
35. Tabung FK, Liu L, Wang W, Fung TT, Wu K, Smith-Warner SA, et al. Association of Dietary Inflammatory Potential with Colorectal Cancer Risk in men and women. JAMA Oncol. 2018;4(3):366-73. https://doi.org/10.1001/ja maoncol.2017.4844.

36. Okura T, Nakamura R, Fujioka Y, Kawamoto-Kitao S, Ito Y, Matsumoto K, et al. Body mass index $\geq 23$ is a risk factor for insulin resistance and diabetes in Japanese people: A brief report. PLoS One. 2018;13(7):e0201052-e.

37. Minihane AM, Vinoy S, Russell WR, Baka A, Roche HM, Tuohy KM, et al. Lowgrade inflammation, diet composition and health: current research evidence and its translation. Br J Nutr. 2015;114(7):999-1012. https://doi.org/10.1017/ S0007114515002093

38. Furukawa S, Fujita T, Shimabukuro M, Iwaki M, Yamada Y, Nakajima Y, et al. Increased oxidative stress in obesity and its impact on metabolic syndrome. J Clin Invest. 2017;114(12):1752-61.

39. Stadler M, Tomann L, Storka A, Wolzt M, Peric S, Bieglmayer C, et al. Effects of smoking cessation on $\beta$-cell function, insulin sensitivity, body weight, and appetite. 2014;170(2):219.

40. Bergman BC, Perreault L, Hunerdosse D, Kerege A, Playdon M, Samek AM, et al. Novel and reversible mechanisms of smoking-induced insulin resistance in humans. Diabetes. 2012;61(12):3156-66. https://doi.org/10.233 7/db12-0418.

41. van der Vaart H, Postma DS, Timens W, Ten Hacken NH. Acute effects of cigarette smoke on inflammation and oxidative stress: a review. Thorax. 2004;59(8):713-21. https://doi.org/10.1136/thx.2003.012468.

42. Gomez-Cabrera M-C, Domenech E, Viña J. Moderate exercise is an antioxidant: upregulation of antioxidant genes by training. Free Radic Biol Med. 2008;44(2):126-31. https://doi.org/10.1016/j.freeradbiomed.2007.02.001.

43. Calder PC, Ahluwalia N, Brouns F, Buetler T, Clement K, Cunningham K, et al. Dietary factors and low-grade inflammation in relation to overweight and obesity. Br J Nutr. 2011;106(S3):S1-S78.

44. DiMenna FJ, Arad AD. Exercise as 'precision medicine' for insulin resistance and its progression to type 2 diabetes: a research review. BMC Sports Science, Medicine and Rehabilitation. 2018:10(1):21.

\section{Publisher's Note}

Springer Nature remains neutral with regard to jurisdictional claims in published maps and institutional affiliations.
Ready to submit your research? Choose BMC and benefit from:

- fast, convenient online submission

- thorough peer review by experienced researchers in your field

- rapid publication on acceptance

- support for research data, including large and complex data types

- gold Open Access which fosters wider collaboration and increased citations

- maximum visibility for your research: over $100 \mathrm{M}$ website views per year

At BMC, research is always in progress.

Learn more biomedcentral.com/submissions 